\title{
Delayed norovirus epidemic in the 2009-2010 season in Japan: potential relationship with intensive hand sanitizer use for pandemic influenza
}

\author{
S. INAIDA ${ }^{1,2 *}$, Y. SHOBUGAWA ${ }^{3}$, S. MATSUNO ${ }^{4}$, R. SAITO $^{3}$ \\ AND H. SUZUKI ${ }^{5}$ \\ ${ }^{1}$ Department of Pharmacoepidemiology, Graduate School of Medicine and Public Health, Kyoto University, \\ Kyoto, Japan \\ ${ }^{2}$ Department of Epidemiology, Graduate School of Medicine, Dentistry and Pharmaceutical Sciences, Okayama \\ University, Okayama, Japan \\ ${ }^{3}$ Division of International Health (Public Health), Niigata University Graduate School of Medical and Dental \\ Sciences, Niigata, Japan \\ ${ }^{4}$ Infectious Disease Surveillance Center, National Institute of Infectious Diseases, Tokyo, Japan \\ ${ }^{5}$ Niigata Seiryo University, Niigata, Japan
}

Received 26 September 2015; Final revision 19 April 2016; Accepted 27 April 2016;

first published online 15 June 2016

\section{SUMMARY}

Norovirus (NoV) epidemics normally peak in December in Japan; however, the peak in the 2009-2010 season was delayed until the fourth week of January 2010. We suspected intensive hand hygiene that was conducted for a previous pandemic influenza in 2009 as the cause of this delay. We analysed the NoV epidemic trend, based on national surveillance data, and its associations with monthly output data for hand hygiene products, including alcohol-based skin antiseptics and hand soap. The delayed peak in the NoV incidence in the 2009-2010 season had the lowest number of recorded cases of the five seasons studied (2006-2007 to 2010-2011). GII.4 was the most commonly occurring genotype. The monthly relative risk of NoV and monthly output of both alcohol-based skin antiseptics and hand soap were significantly and negatively correlated. Our findings suggest an association between hand hygiene using these products and prevention of NoV transmission.

Key words: Alcohol, hand hygiene, hand soap, norovirus, sentinel surveillance.

\section{INTRODUCTION}

Norovirus (NoV) is a major cause of viral gastroenteritis, with epidemics occurring in the autumn to winter seasons, from about September to February in Japan and other temperate countries. The recent emergence of a new GII.4 variant, 2006b [1], resulted in the largest NoV epidemic to be detected in the history of national surveillance of infectious diseases since its launch in the 1980s. At that time, public awareness of NoV

\footnotetext{
* Author correspondence: Dr S. Inaida, Yoshidakonoe-cho, Sakyoku, Kyoto City, Kyoto 606-8501, Japan.

(Email: inaida.shinako.7v@kyoto-u.ac.jp)
}

developed markedly, and governmental guidelines for hygiene were shared [2].

NoV transmission is thought to occur primarily through three routes: person-to-person, foodborne, and waterborne. Person-to-person transmission occurs directly through the faecal-oral route by ingestion of aerosolized vomitus or indirect exposure via fomites or contaminated environmental surfaces [3]. In addition to rigorous disinfection of contaminated surfaces using sodium hypochlorite (200 ppm) antiseptic and preventing food handling by people suspected of being infected, hand hygiene is thought to be the most important method to prevent transmission [4]. However, tests have resulted in different levels of effectiveness with 
alcohol-based hand sanitizers to reduce NoV on hands [5-14]. Washing hands with hand soap is also thought to be effective for prevention [7, 15].

In the 10 years since 2002, recurrent epidemic peaks in Japan mostly occurred in December; however, the peak in the 2009-2010 season was delayed until the fourth week in January 2010. This delay represented a new event historically in Japan; therefore, we expected that an associated background factor affected the epidemic.

Coincidentally, a pandemic influenza was caused by the pandemic $\mathrm{A}(\mathrm{H} 1 \mathrm{~N} 1) 2009$ virus in the 2009-2010 season, which was first observed in Mexico and soon became prevalent worldwide in March 2009 [16, 17]. The World Health Organization announced a phase 6 pandemic on 11 June 2009. In Japan, the first case of the pandemic virus was detected on 9 May 2009, and the epidemic began at the beginning of August 2009, which was 3-4 months earlier than that of seasonal influenza. On 22 May 2009, the Ministry of Health, Labour, and Welfare in Japan announced the following countermeasures for the pandemic [18]: preventive measures at a personal level such as overall hygienic behaviour (gargling, washing hands, wearing masks), vaccination, and avoiding contact with infected people as well as public health measures such as placing liquid alcohol-based hand sanitizers at the entrance to public buildings [19]. All of the schools, workplaces, stores, and public facilities immediately placed these sanitizers at their entrances, and alcohol-based hand hygiene was the most intensively used ever at the national level in addition to the previously mentioned personal preventive measures.

We suspected that the intensive hand hygiene measures for pandemic influenza influenced the delay of the NoV epidemic in the 2009-2010 season. Therefore, this study aimed to determine if personal hand hygiene measures, such as alcohol-based hand hygiene or hand soap, were associated with NoV for the five seasons from 2006-2007 to 2010-2011.

\section{METHODS}

\section{NoV surveillance data}

We used the national sentinel gastroenteritis surveillance data from the National Epidemiological Surveillance of Infectious Diseases (NESID) of the National Institute of Infectious Diseases for the period of September to February, which covered about the 36th to 8th epidemiological weeks of the epidemic season of the diseases [20], within the last five seasons from 2006-2007 to 2010-2011.
The NESID data include the weekly number of cases of gastroenteritis from $\sim 3000$ paediatric clinics across the country, and the level of epidemic on an annual basis has been monitored for more than 40 years using the weekly number of cases per sentinel site. The number of sentinel clinics is defined by population size within each health centre's sector [21]. The data are collected by physicians who report a diagnosis of gastroenteritis to the regional health centres; the diagnosis is based on the symptoms of diarrhoea, vomiting, and acute abdominal pain (for an infant, abdominal pain might not be clear). The regional health centres enter the data into NESID through an online system on a weekly basis. The data include both sporadic cases and outbreaks [21].

Although there are causes of gastroenteritis other than NoV, such as bacteria and other viruses, NoV is diagnosed using rapid test kits or polymerase chain reaction (PCR) methods at laboratories. For laboratory testing, specimens were routinely collected from about $10 \%$ of inpatients and outpatients at hospital admission who were diagnosed at the sentinel clinics. The results of viral genotyping from real-time PCR were available at most prefectural institutes and reported to NESID. We examined the proportion of each viral genotype for 2006-2011.

We observed the trend in epidemic curves using the surveillance data. Statistical indices calculated from the weekly incidences were also examined. The threshold for an NoV epidemic was set at $4 \cdot 0$ cases to examine the timing of a primary increase in cases because in the last 10 years, NoV sentinel cases continued to increase after the number of sentinel cases reached $>4 \cdot 0$ until the peak of the epidemic [20]. As an example, the epidemic curve of pandemic influenza from the surveillance data is shown in Supplementary Figure S1. Pearson correlations of weekly sentinel data were compared between the 2006-2007 season and the four seasons between 2007-2008 and 2010-2011 using SPSS v. 10 (SPSS Inc., USA). The monthly relative risk of NoV was calculated for the 2009-2010 season.

\section{Output data for hygiene products}

To consider the level of hand hygiene behaviour from either hand soap (total of medicated soap and liquid hand soap) or alcohol against pandemic influenza during the 2009-2010 season, we used yearly and monthly output data. The data were obtained from the Statistics of Production of Chemical Industry of the 


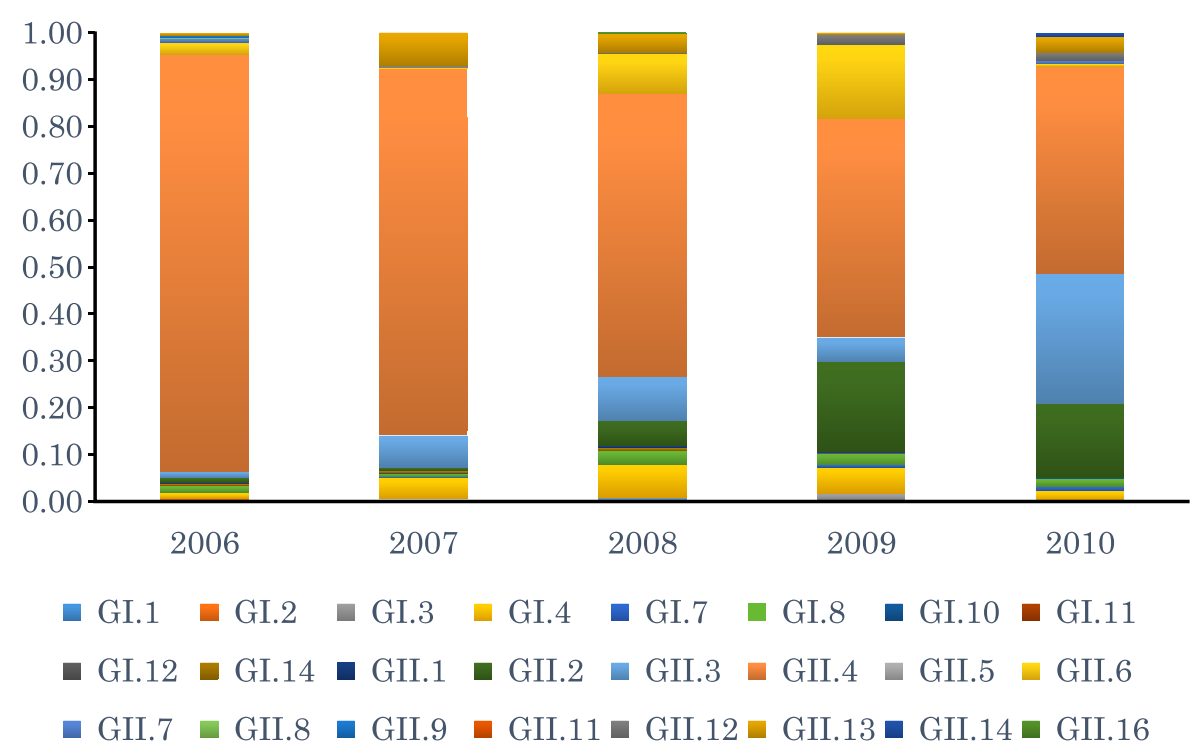

Fig. 1. Distribution of norovirus genotypes (2006-2010). The data were obtained from the Infectious Agents Surveillance Report (IASR) (http://www.nih.go.jp/niid/en/).

Ministry of Economy and Industry in Japan for liquid hand soap and the Statistics of Production by Pharmaceutical Industry of the Ministry of Health, Labour, and Welfare in Japan for both medicated soap and skin antiseptics for non-medication use. The skin antiseptics were assumed to mainly consist of alcohol-based liquid hand sanitizer. Pearson correlations were analysed between these outputs and the monthly relative risk of NoV in the 2009-2010 season. To examine the possible effect, the yearly output of masks (medical, industrial, and domestic uses) is shown in Supplementary Figure S2b.

\section{Meteorological data}

Because the climatic condition is thought to affect NoV epidemics $[20,22]$, we sought to determine if there was any variation or change in the meteorological trends during the study period. Monthly average temperatures, relative humidity, and rainfall were obtained from the web-based source of the Japan Meteorological Agency and compared between a recent 12-year period (20002011) and 2009 (Supplementary Fig. S3).

\section{Ethical standards}

The authors assert that all procedures contributing to this work comply with the ethical standards of the relevant national and institutional committees on human experimentation and with the Helsinki Declaration of 1975 , as revised in 2008.

\section{RESULTS}

\section{Predominant norovirus genotype}

GII.4 was the most frequently occurring genotype in all five seasons based on the PCR tests (Fig. 1). However, the rate of GII.4 within the detected genotypes declined from $88.9 \%$ in the $2006-2007$ season to $46.7 \%$ in the 2009-2010 season and $44 \cdot 2 \%$ in the 2010-2011 season. GII.3 increased from $1 \cdot 3 \%$ in the 2006-2007 season to $27 \cdot 8 \%$. GII. 2 was the second most frequently occurring genotype in the 2009-2010 season, followed by GII.6.

\section{Peak week and correlations between seasons}

The peaks of the NoV epidemic appeared in weeks 49-51 in the observed seasons, except the 2009-2010 season when the peak was delayed until week 4 in January 2010 (Fig. 2 and Table 1). Although Figure 2 shows only five seasons, the epidemic peak during the previous 10 years, i.e. since 2002, also occurred at a similar time, in weeks 50-52 [23].

The incidence of NoV (number of cases per sentinel site) during the peak week was highest at 22.81 cases in the 2006-2007 season and lowest at 14.32 cases in the 2009-2010 season. The average weekly incidence was highest in the 2006-2007 season (9.18 cases); the average weekly incidences were similar between the 20092010 (6.19 cases) and 2008-2009 (6.72 cases) seasons, during which the incidences were relatively low and the epidemic seasons were smaller than the other observed seasons. The median value was lowest in the 


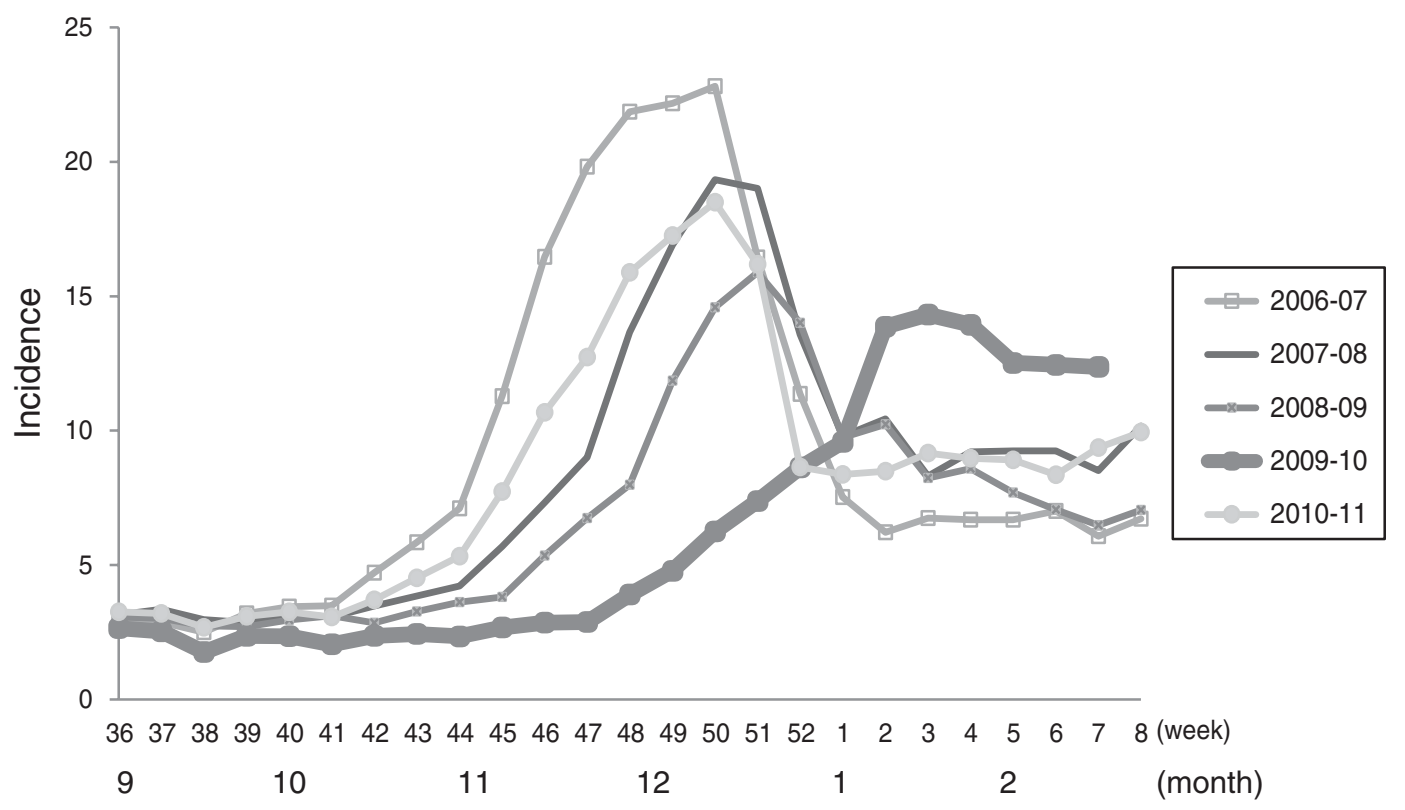

Fig. 2. Weekly epidemic curves of norovirus cases in the 2006-2007 to 2010-2011 seasons. The data show the number of cases per sentinel site obtained from the National Epidemiological Surveillance of Infectious Diseases (NESID) in Japan (http://www.nih.go.jp/niid/en/survaillance-data-table-english.html).

Table 1. Trends in norovirus epidemics in Japan, based on surveillance data

\begin{tabular}{lccccr}
\hline \hline Season & $2006-2007$ & $2007-2008$ & 2008-2009 & 2009-2010 & 2010-2011 \\
\hline Average & $9 \cdot 18$ & $8 \cdot 21$ & $6 \cdot 72$ & $6 \cdot 19$ & $8 \cdot 44$ \\
Median & $6 \cdot 69$ & $8 \cdot 31$ & $6 \cdot 49$ & $3 \cdot 91$ & $8 \cdot 49$ \\
Min & $2 \cdot 50$ & $2 \cdot 87$ & $2 \cdot 71$ & $1 \cdot 77$ & $14 \cdot 32$ \\
Max & $22 \cdot 81$ & $19 \cdot 33$ & $15 \cdot 88$ & $154 \cdot 74$ & $210 \cdot 96$ \\
Total & $229 \cdot 49$ & $205 \cdot 13$ & 469 & 42 \\
Epidemic threshold* & 42 & 43 & 51 & 4 & 50 \\
Epidemic peak* & 50 & 50 & 7 & 8 & 8 \\
Time to peak week after & 8 & 7 & & & \\
threshold (weeks) & & & & & \\
\hline \hline
\end{tabular}

* Epidemiological week.

2009-2010 season (3.91 cases) and ranged from $6 \cdot 49$ to 8.49 cases in the other seasons. The total incidence between the 36th and 8th weeks was highest in the 2006-2007 season (229.49 cases), followed by the 2010-2011 (210.96 cases), 2007-2008 (205.13 cases), 2008-2009 (167.95 cases), and 2009-2010 (154.74 cases) seasons. The weekly increase in NoV cases was also lower in the 2009-2010 season, at $0 \cdot 1$ cases per week, compared to an average 1.9 cases per week in the other seasons. A relatively more rapid increase of NoV incidence in the 2009-2010 season began after week 48, when influenza peaked (Supplementary Fig. S1). The time between the threshold week $(\geqslant 4 \cdot 0$ recorded cases) and the peak week were similar, at
7-8 weeks, in all of the observed seasons. After the peak of the epidemic, the incidence of NoV decreased sharply, except during the 2009-2010 season.

The correlation coefficients of the weekly sentinel data between the 2006-2007 season and the following four seasons (2007-2008 to 2010-2011) were 0.793 $(P<0.01), 0.651 \quad(P<0.01),-0.078(P=0.71)$, and $0.908(P<0.01)$, respectively.

\section{Output data of skin hygiene products, relative risk, and meteorological trend}

The monthly output of skin antiseptics (for nonmedication use) increased until October 2009, when 
(a)

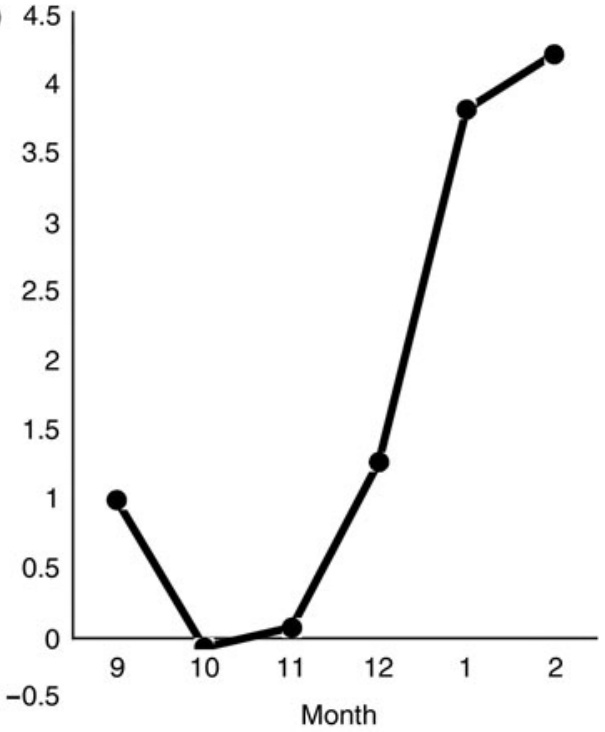

(b)

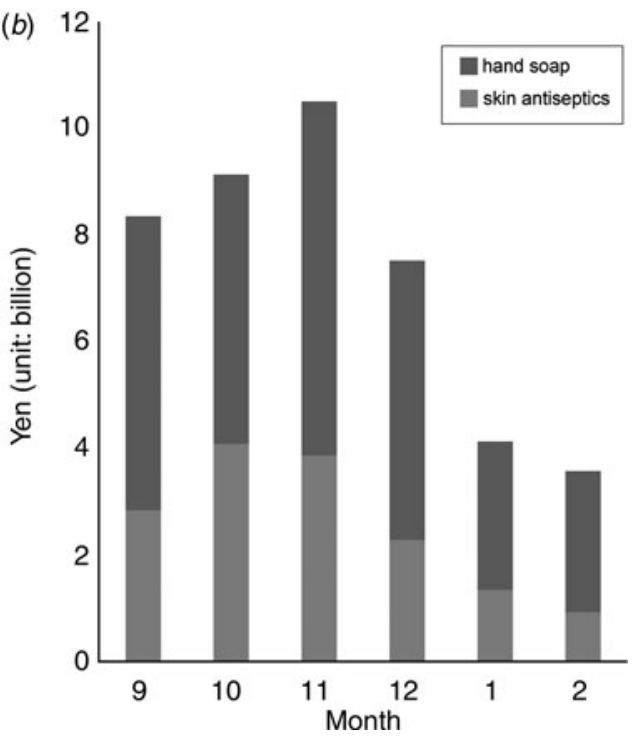

Fig. 3. (a) Monthly relative risk of norovirus based on the monthly average incidence from sentinel data and $(b)$ the monthly output of skin hygiene products. The monthly total output (units: billion yen) of skin antiseptics for non-medication use (representing alcohol-based liquid hand sanitizer) and hand soap (total of liquid soap and medicated soap) between September 2009 and February 2010. The data were obtained from the Statistics of Production of Chemical Industry (liquid hand soap) of the Ministry of Economy and Industry in Japan [http://www. meti.go.jp/statistics/ (in Japanese)] and from the Statistics of Production by Pharmaceutical Industry (medicated soap) of the Ministry of Health, Labour and Welfare in Japan [http://www.mhlw.go.jp/topics/yakuji.html (in Japanese)].

it was 1.44 times higher than that in September 2009, which was also when the incidence of pandemic influenza increased (Fig. 2, Supplementary Fig. S1). The skin antiseptic output decreased slightly in
November but remained similarly high and then rather rapidly decreased in December (Fig. 3b).

The monthly output of hand soap showed a small decrease in October and then increased in November, when it was 1.20 times higher than that in September. It started decreasing in December when the peak of pandemic influenza ended and the increase in NoV began (Fig. 3a). The correlation coefficients between the relative risk of NoV and skin antiseptic and hand soap outputs were -0.97 $(P<0.01)$ and $-0.93(P<0.01)$, respectively.

The yearly total output of skin antiseptics reached 21.7 billion yen in 2009 , which was about 4.23 times higher than the average output of the recent three years (Supplementary Fig. S2). The yearly output of hand soap was 1.66 times higher than that of the average output of the recent three years. The annual output of masks also sharply increased in 2009, with the highest increase for domestic use (Supplementary Fig. S2).

The average temperature, relative humidity, and rainfall in 2009 were very similar to those of each of the previous 12 years (Supplementary Fig. S3a, b).

\section{DISCUSSION}

The delayed epidemic peak in the 2009-2010 season represented the first occurrence in the last 10 years. Although the proportion of the GII.4 NoV genotype decreased over the five seasons in the present study, it remained the genotype with the highest proportion throughout all of the seasons. The GII.4 variant 2006b was dominant in the 2006-2007 season and predominant in the 2007-2008 and 2008-2009 seasons [24].

It was not certain whether a new viral variant had emerged or whether other factors, such as immunity development, affected the timing of the epidemic peak in the 2009-2010 season. Despite the significant correlations between the sentinel data from the 2006 2007 season with those from the three later seasons, the data from the 2006-2007 season were not significantly correlated with those from the 2009-2010 season. There were also no major differences in meteorological trends. Furthermore, the correlation between the monthly relative risk of NoV and output of skin antiseptics and hand soap suggested that hand hygiene using alcohol-based liquid hand sanitizer and washing hands with hand soap could have prevented NoV transmission and therefore possibly delayed the epidemic season.

During the pandemic period, the media updated the public regarding the epidemic level, which was 
monitored by sentinel surveillance. After the peak of the pandemic, less intensive use of alcohol-based sanitizer for hand hygiene or less frequent hand washing would have occurred, as indicated by the decrease in those outputs. As with the recurrence of NoV epidemic peaks in other seasons, the NoV epidemic seems to be climate dependent. However, it is not certain why the NoV incidence in 2009-2010 continued increasing after the peak of the pandemic when the usual seasonal timing had passed. More interestingly, the delayed peak of NoV was at a similar level as 2008-2009, which was the smallest epidemic season in the observed seasons, and after the delayed peak week the incidence did not show an immediate decrease. Thus, the total incidences were also similar between the 2009-2010 and 2008-2009 seasons. However, the overall statistical indices (i.e. epidemic timing, time to peak week after the threshold, and total incidence) suggested that the NoV epidemics recurred in a similar trend over time, indicating a general impact or general characteristics of NoV activity in the infection. Further studies to clarify the background of the epidemic recurrence are warranted.

Meanwhile, both alcohol-based sanitizer and hand soap have been extensively used as a general hygiene measure at hospitals and in homes in Japan, even before the pandemic season. The increase in these outputs during the pandemic season was considered as a result of increased frequency of use for hand hygiene. Alcohol-based sanitizer was primarily placed and used at the entrances of public buildings, and washing hands was usually performed after using the bathroom or before handling food; however, the order in which both or either alcohol-based sanitizer and hand soap are used for hygiene purposes remains unknown.

Although further clarification is warranted to determine the efficacy of alcohol-based sanitizers and hand soap for NoV, our findings suggest an association between hand hygiene conducted using alcohol-based products and hand soap and prevention of NoV transmission, resulting in decreased epidemic levels. A limitation of the available output data was the lack of product formulation and other data such as contact time or comparison of different sanitizer products by setting, which could affect preventive efficacy $[7,11,15]$.

There could have been a time lag between the recording of the output and the use of those hygiene products. However, it is likely that the stocks of such seasonal hygiene products are limited.
The output data regarding masks indicated an increased use by individuals and suggested that the use of masks might also have decreased the transmission of airborne infections. However, the data were only available yearly and not monthly. During the pandemic, people would have used more of the hygiene measures suggested as pandemic countermeasures, such as cleaning of surfaces, opening windows to prevent virus spread, and avoiding crowded places [17]. Likewise, more prevention methods would have been performed in hospitals and homes. Thus, there would have been less NoV transmission compared to the other seasons. Other climatic conditions might have also affected the epidemic.

A vaccine for human NoV has not been developed and is not available owing to a failure in culturing the virus. Thus, accomplishing hand hygiene is probably the most important measure for reducing personto-person spread of NoV and preventing an epidemic. An effective set of control measures should be sought in future studies.

\section{SUPPLEMENTARY MATERIAL}

For supplementary material accompanying this paper visit http://dx.doi.org/10.1017/S0950268816000984.

\section{ACKNOWLEDGEMENTS}

This study was supported by Kyoto University Startup Grant from Kyoto University.

\section{DECLARATION OF INTEREST}

None.

\section{REFERENCES}

1. Motomura K, et al. Identification of monomorphic and divergent haplotypes in the 2006-2007 norovirus GII/4 epidemic population by genomewide tracing of evolutionary history. Journal of Virology 2008; 82: 1124711262.

2. Ministry of Health, Labour and Welfare. Q\&A about norovirus in Japan [in Japanese] (http://www.mhlw.go. jp/topics/syokuchu/kanren/yobou/040204-1.html). Accessed 12 December 2012.

3. Marks PJ, et al. Evidence for airborne transmission of Norwalk-like virus (NLV) in a hotel restaurant. Epidemiology \& Infection 2000; 124: 481-487.

4. Division of Viral Diseases, National Center for Immunization and Respiratory Diseases, Centers for Disease Control and Prevention. Updated norovirus outbreak management and disease prevention guidelines report. 
Morbidity and Mortality Weekly Report. Recommendations and Reports 2011; 60: 1-18.

5. Friesema IH, et al. Norovirus outbreaks in nursing homes: the evaluation of infection control measures. Epidemiology \& Infection 2009; 137: 1722-1733.

6. Shimizu-Onda Y, et al. The virucidal effect against murine norovirus and feline calicivirus as surrogates for human norovirus by ethanol-based sanitizers. Journal of Infection and Chemotherapy 2013; 19: 779-781.

7. Sattar SA, Ali M, Tetro JA. In vivo comparison of two human norovirus surrogates for testing ethanol-based handrubs: the mouse chasing the cat! PLoS ONE 2011; 6: e17340.

8. Cheng VC, et al. Prevention of nosocomial transmission of norovirus by strategic infection control measures. Infection Control \& Hospital Epidemiology 2011; 32: 229-237.

9. Belliot G, et al. Use of murine norovirus as a surrogate to evaluate resistance of human norovirus to disinfectants. Applied and Environmental Microbiology 2008; 74: 3315-3318.

10. Steinmann J, et al. Comparison of virucidal activity of alcohol-based hand sanitizers versus antimicrobial hand soaps in vitro and in vivo. Journal of Hospital Infection 2012; 82: 277-280.

11. Steinmann J, et al. Virucidal activity of 2 alcohol-based formulations proposed as hand rubs by the World Health Organization. American Journal of Infection Control 2010; 38: 66-68.

12. Liu P, et al. Effectiveness of liquid soap and hand sanitizer against Norwalk virus on contaminated hands. Applied and Environmental Microbiology 2010; 76: 394-399.

13. Nowak $\mathbf{P}$, et al. Measurement of the virolysis of human GII.4 norovirus in response to disinfectants and sanitisers. Journal of Virological Methods 2011; 174: 7-11.

14. Cheng VC, et al. Successful control of norovirus outbreak in an infirmary with the use of alcohol-based hand rub. Journal of Hospital Infection 2009; 72: 370-371.
15. Tuladhar E, et al. Reducing viral contamination from finger pads: handwashing is more effective than alcoholbased hand disinfectants. Journal of Hospital Infection 2015; 90: 226-234.

16. Centers for Disease Control and Prevention (CDC). Outbreak of swine-origin influenza A (H1N1) virus infection-Mexico, March-April 2009. Morbidity and Mortality Weekly Report 2009; 58: 467-470.

17. Ministry of Health, Labour, Welfare. Report of the review meeting on measures against pandemic influenza (A/H1N1) [in Japanese] (http://www.mhlw.go.jp/ bunya/kenkou/kekkaku-kansenshou04/info_local.html). Accessed 12 October 2012.

18. Ministry of Health, Labour and Welfare. Announcement for the countermeasures to the pandemic in Japan [in Japanese] (http://www.mhlw.go.jp/kinkyu/kenkou/ influenza/dl/090522-03a.pdf). Accessed 2 March 2012.

19. SteelFisher GK, et al. Public response to the 2009 influenza A H1N1 pandemic: a polling study in five countries. Lancet Infectious Diseases 2012; 12: 845-850.

20. Inaida $\mathbf{S}$, et al. The south to north variation of norovirus epidemics from 2006-07 to 2008-09 in Japan. PLoS ONE 2013; 8: e71696.

21. Taniguchi K, et al. Overview of infectious disease surveillance system in Japan, 1999-2005. Journal of Epidemiology 2007; 17: S3-S13.

22. Lopman B, et al. Host, weather and virological factors drive norovirus epidemiology: time-series analysis of laboratory surveillance data in England and Wales. PLoS ONE 2009; 4: e6671.

23. National Institute of Infectious Disease. Infectious gastroenteritis cases reported per sentinel weekly (http://www.nih.go.jp/niid/en/survaillance-data-tableenglish.html) Accessed 12 December 2012.

24. Motomura K, et al. Divergent evolution of norovirus GII/4 by genome recombination from May 2006 to February 2009 in Japan. Journal of Virology 2010; 84: 8085-8097. 